\title{
WHY IS THE HI SCALE HEIGHT CONSTANT WITH GALACTOCENTRIC RADIUS?
}

\author{
A. FERRARA \\ Osservatorio Astrosifico di Arcetri \\ Largo E. Fermi, 550125 Firenze, Italy
}

\section{Introduction}

The vertical distribution of neutral hydrogen has been studied over the last three decades (Schmidt 1957; Baker \& Burton 1979; Lockman 1984; Savage $\&$ Massa 1987). The best fit curve to the HI $z$-distribution in the solar neighborhood has a FWHM of about $230 \mathrm{pc}$, and it extends appreciably beyond 500 pc (Dickey \& Lockman 1990).

The support of the Galactic HI layer in the Galactic gravitational potential may be provided by bulk motions of the gas. Using a hydrostatic approach, Lockman \& Gehman (1991) have demonstrated elegantly that, in order to reproduce the observed distribution, a number of isothermal components, with different one-dimensional velocity dispersion $\sigma$, must be considered. In these terms, a population of "very fast" $\left(\sigma_{f} \geq 25 \mathrm{~km} \mathrm{~s}^{-1}\right)$ clouds seems to be necessary to explain the high- $z$ tail of the distribution.

A population of fast clouds appears to be a basic ingredient of any model in which the vertical support of the HI is due to turbulent pressure alone, mainly to account for the extended tail of the distribution. The values of $\sigma_{f}$ discussed above are certainly marginally sufficient to account for the observed HI scale height and distribution at $\varpi=\varpi_{\odot}$, but they are still quite controversial. In an analysis of the Galactic hydrostatic equilibrium, Bloemen (1987) uses a single value $\sigma=9 \mathrm{~km} \mathrm{~s}^{-1}$ for the HI dispersion; Boulares \& Cox (1990) adopt a two population HI cloud model with $\sigma_{s}=$ $9 \mathrm{~km} \mathrm{~s}^{-1}$ and $\sigma_{f}=14.3 \mathrm{~km} \mathrm{~s}^{-1}$ for slow and fast clouds, respectively. Supernovæ are generally considered to represent the major energy source for turbulent motions; nevertheless, an efficient conversion of the explosive energy injection into turbulent pressure relies on a high porosity parameter of the ISM, Q, which is far cry from being determined (McKee, 1990). 
In addition, the mechanisms accelerating the fast clouds - e.g. supernova shocks- are also likely to partially destroy them through fragmentation and evaporation (Klein, McKee \& Colella, 1990). The two previous remarks underline that the origin, and perhaps even the very existence, of a class of very fast clouds $\left(\sigma_{f} \geq 25 \mathrm{~km} \mathrm{~s}^{-1}\right)$ is troublesome. This complication can put pure turbulent pressure HI support models into some difficulty.

Apart from the large $z$-extension of the HI, its scale height is apparently constant of over a large range of galactocentric radii, $\varpi$, despite a sensible variation of the gravitational potential in the same range (Dickey \& Lockman 1990; Heiles 1991). Also, the shape of the vertical HI distribution remains almost the same in $4 \leq \varpi \leq 8 \mathrm{kpc}$.

In this paper we stress the relevance of the Galactic radiation field to account for the HI distribution in the Galaxy; in addition we present a simple model for the origin of turbulent motions that reproduces many of the features of the local, turbulent ISM.

\section{Radiative/Turbulent Support of HI}

In the following we present briefly the model for the radiative/turbulent support of the HI; a more extended description can be found in Ferrara (1993). Dust grains located at an optical depth $\tau<1$ in an interstellar cloud immersed in a radiation field, feel a radiation pressure which depends on the field flux, F, and on the grain radiation pressure coefficient, $Q_{p}$ (Franco et al. 1991; Ferrara 1993). The momentum transferred by the photons to the grains can be transmitted to the cloud gas provided that the dustgas coupling, mainly represented by viscous and Coulomb drag, is strong enough. Hence, the requirement for a cloud to be effectively photolevitated by the radiation field is that its column density must be in the interval $10^{19} \leq N_{H} \leq 5 \times 10^{20} \mathrm{~cm}^{-2}$.

If the photon field is anisotropic, the cloud receives a net acceleration which depends on the ratio $\gamma(r)$ between the average energy flux at $z$ directed towards the disk, $F_{\text {down }}$, and the one directed upwards, $F_{u p}$

$$
\gamma(r)=\frac{F_{\text {down }}^{\prime}}{F_{u p}}=\frac{\int_{z}^{\infty} F(z) d z}{\int_{-\infty}^{z} F(z) d z}=\frac{1-W(r)}{1+W(r)},
$$

where $r=z / H_{\star}, W(r)$ and $H_{\star}$ are the distribution and scale height of the disk stars, respectively. Besides the average interstellar radiation field, a contribution to the radiation pressure on the cloud due to stellar clusters, $P_{c l}$ is also included. The total radiation pressure, $P_{r a d}$, therefore, can be written as

$$
P_{r a d}=P_{a v}+P_{c l}=\left\{\frac{Q_{p} F}{c}\left[\frac{1-\gamma(r)}{1+\gamma(r)}\right]+\frac{Q_{p}\langle L\rangle}{4 \pi z^{2} c}\right\}\left(1-e^{-\tau_{c}}\right)
$$


where $F=F_{u p}+F_{\text {down }}$ is the total power per unit surface from one face of the disk, $\langle L\rangle$ is the average cluster luminosity, and $\tau_{c}$ is the total optical depth of the cloud. A detailed evaluation of the galactocentric dependence of the parameters entering in eq. (2) is given by Ferrara (1993).

The radiative force adds to the gravitational one acting on the clouds, resulting in an "effective" potential $\phi_{e}$. The expression for $\phi_{e}$ is

$$
\phi_{e}(\varpi, z)=\int_{0}^{z} K_{z}(\varpi, z) d z-\int_{0}^{z} \frac{P_{r a d}(\varpi, z)}{N \mu} d z=\int_{0}^{z} K_{z}(\varpi, z) d z,
$$

where $N$ is the cloud total column density along the $z$-axis, $\mu$ is the mean mass per gas particle, and $K_{z}(z)$ is the gravitational acceleration (Kuijken \& Gilmore 1989).

The density distribution $n(z)$ can be obtained by solving the hydrostatic equilibrium equation

$$
\nabla p=-\rho \nabla \phi_{e} .
$$

If one admits that the pressure is provided solely by gas motions, then $p=\rho \sigma^{2}$; supposing also that the HI can be approximated as the sum of the "fast" and "slow" isothermal components, eq. (4) has the solution

$$
\rho(z)=\sum_{j=f, s} \rho_{0, j} \exp \left[-\phi_{e}(z) / \sigma_{j}^{2}\right]
$$

where the indexes $f$ and $s$ stand for fast and slow, respectively, and $\rho_{0, j}$ is the density at midplane. The total density profiles obtained from eq. (5) at different $\varpi$ have been compared with the average observed profile given by Dickey \& Lockman (1990) for all galactocentric radii considered (Ferrara 1993). The fits are remarkably good in the entire range $4 \leq \varpi \leq 8.5 \mathrm{kpc}$ assuming a slightly increasing value for $\sigma_{j}$ (both for the fast and the slow clouds) towards the Galactic center: for $\varpi=\varpi_{\odot}=8.5 \mathrm{kpc}\left(\sigma_{f}=13.0, \sigma_{s}=\right.$ $5.0) \mathrm{km} \mathrm{s}^{-1}$, whereas for $\varpi=4.0 \mathrm{kpc}\left(\sigma_{f}=22.0, \sigma_{s}=8.0\right) \mathrm{km} \mathrm{s}^{-1}$.

We conclude that:

- The vertical support of the galactic HI may be completely provided by turbulence- $e . g$., bulk motions of the gas- without requiring any additional pressure due to magnetic fields and cosmic rays when the average Galactic and cluster radiation fields acting on the clouds are included. The photolevitation mechanism tends to create a lower "effective" gravitational potential, as described above.

- The observed constancy of the HI scale height with $\varpi$ is naturally explained by the model in terms of the similar radial dependence of the average luminosity and mass distribution found in spiral galaxies and, in particular, in the Milky Way. 
- The increase in the velocity dispersion of the fast clouds implied by the fit parameters towards the Galactic center closely resembles the global distribution of the HII regions in the disk. This is also consistent with the suggestion by Dickey \& Lockman (1990) that the dispersion may increase toward the inner Galaxy.

In the following we investigate the origin and the energy budget of the turbulent motions in the ISM.

\section{Sources of Turbulent Energy}

The kinetic energy stored in turbulent motions can be estimated from observational data to be $E_{k} \sim 6 \times 10^{51} \mathrm{ergs}$ (Lockman \& Gehman 1991). The most plausible sources of this energy are supernova explosions, which are likely to occur in a correlated manner, producing supershells. Correlated supernovæ can inject kinetic energy into the ISM at a rate

$$
\dot{E}_{S N} \sim 10^{39}\left(\frac{N_{s s}}{10^{3}}\right)\left(\frac{E_{0}}{10^{51} \mathrm{erg}}\right)\left(\frac{\Delta \tau}{2 \times 10^{5} \mathrm{yr}}\right)\left(\frac{\eta}{0.01}\right) \mathrm{erg} \mathrm{s}^{-1},
$$

where $N_{s s}$ is the number of supershells in the disk, $E_{0}$ is the total energy of a supernova, $\Delta \tau$ is the time interval between explosions, and $\eta$ is the efficiency of the conversion of $E_{0}$ into kinetic motions. According to this estimate, the supply time for the turbulent energy is roughly $2 \times 10^{5} \mathrm{yr}$; in order to have a steady-state the decay time of the turbulent spectrum must be of the same order. Our approach to determine this quantity is to use the local interstellar medium (LISM). Since accurate measurements are possible for this environment, it represents an ideal laboratory to understand the decay of turbulence.

\subsection{A FEW POINTS ABOUT THE LOCAL BUBBLE}

The Local Bubble (LB) is an oval-shaped cavity filled with hot $\left(T_{h} \sim\right.$ $\left.10^{6} \mathrm{~K}\right)$, tenuous $\left(n_{h} \sim 5 \times 10^{-3} \mathrm{~cm}^{-3}\right)$ gas surrounding the sun. Its origin probably requires a supernova (SN) explosion that occurred close to the sun $\sim 2-10 \times 10^{5}$ yr ago (Cox \& Reynolds 1987). This hypothesis has found strong support after the identification of the $\gamma$-ray source Geminga with the $\mathrm{x}$-ray source $1 \mathrm{E} 0630+178$ (Bignami et al. 1983), recently discovered to be a pulsar by ROSAT observations. The age of the pulsar, as derived from its period, is $3.2-3.4 \times 10^{5} \mathrm{yr}$ (Gehrels \& Chen 1993); its distance from the sun is estimated to be $40-400$ pc. On this basis, Gehrels \& Chen conclude that the origin of the LB can well be ascribed to the Geminga supernova explosion.

In addition to the hot gas, several low-density HI clouds are interspersed in the ionized medium; the sun itself is located at the edge of the so-called 
Local Cloud (LC). A likely possibility is that these structures are remnants of clouds that have been engulfed by the interstellar shock produced by the explosion, as predicted by the McKee \& Ostriker (MO:1977) model. During this process, turbulence generation is expected as a result of noncoherent overlapping of the secondary shocks created by the interaction of the primary one with the clouds. The very complex velocity structure seen in the LC (Lallement et al. 1986) is suggestive of a turbulent pattern and of the fact that the cloud has been shocked (Bruhweiler \& Vidal-Madjar 1987).

Recent absorption line studies (Linsky et al. 1993) have exploited the high-resolution, high signal-to-noise data from the Hubble Space Telescope Goddard High-Resolution Spectrometer to observe the line of sight toward the star Capella, located $13.2 \mathrm{pc}$ away from the sun. In brief, the results of interest here are the determination (for the first time based on a direct analysis of the absorption line profiles rather than on equivalent widths) of an average hydrogen density $n_{H I}=0.047 \mathrm{~cm}^{-3}$, a temperature $T=$ $7000 \pm 200 \mathrm{~K}$ and a turbulent velocity $\xi=1.66 \pm 0.03 \mathrm{~km} \mathrm{~s}^{-1}$. This last result is noticeably interesting, as $\xi$ is substantially lower than the values inferred for the mean cloud velocity dispersion in the general ISM (see above).

\subsection{EVOLUTION OF THE TURBULENT SPECTRUM IN THE LOCAL BUBBLE}

In the following we present a model for the evolution and decay of the turbulent spectrum injected by a SN explosion in the LB. The aim is to reproduce the low velocity dispersion observed locally in a self-consistent manner. The interested reader may find a more extended presentation in Ferrara (1994).

Let's assume homogeneous, isotropic turbulence: this approximation is rather crude but still useful for the present aim; in addition, the results can be used at least as a guide when discussing compressible turbulence.

Next, we introduce a spectral representation of the velocity field in the fluid: the energy contained in eddies with wavenumber between $k$ and $k+d k$ is given by $\rho E(k, t) d k$, where $\rho$ is the density of the fluid and $E(k, t)$ is the (tridimensional) spectrum of the turbulence.

To study the evolution of the spectrum when the energy source is turned off (i.e., after the supernova explosion) it is necessary to take into account the dynamic equation governing the time evolution of $E(k, t)$. In its integral form this equation reads (Batchelor 1986)

$$
\frac{\partial}{\partial t} \int_{0}^{k} d k E(k, t)=\int_{0}^{k} d k F(k, t)-2 \nu \int_{0}^{k} d k k^{2} E(k, t)
$$


here $F(k, t)$ is the energy-transfer function, $\nu=\mu / \rho$ is the kinematic viscosity. The physical meaning of eq.(7) is transparent and can be understood in the following way. The lhs term describes the change in the kinetic energy and the dissipation occurring in large eddies with wavenumber less than $k$. The flow of energy from large eddies is split in two different contributions: part of it is transmitted in form of kinetic energy to smaller eddies, and part is directly dissipated into heat through viscosity. The first two terms on the rhs describe these two processes, respectively.

A complete solution of the dynamic equation in its general form has not yet been found and therefore it is necessary to postulate an explicit form of $F(k, t)$; this is usually done on the basis of some physical/dimensional arguments. We have adopted the formulation proposed by Kovasznay (1948),

$$
\int_{0}^{k} d k F(k, t)=-\alpha E^{3 / 2}(k, t) k^{5 / 2},
$$

since this form automatically satisfies the condition of a local interaction in every region of the spectrum (Ferrara 1994). Given the adopted form of the transfer function it follows that eq.(7) can be more fruitfully written in its differential form

$$
\frac{\partial E(k, t)}{\partial t}=-\alpha \frac{\partial}{\partial k}\left(E^{3 / 2} k^{5 / 2}\right)-2 \nu k^{2} E(k, t),
$$

where $\alpha$ is a constant of the order of unity.

Eq.(9) completely describes the evolution of the turbulent spectrum, provided the appropriate boundary and initial conditions are specified. A solution of eq. (9) is found using the method of characteristics. Introducing the auxiliary variable $\Psi(k, t)$, such that $E(k, t)=\Psi^{2}(k, t) k^{-5 / 3}$ we obtain

$$
\frac{2}{\Psi(k, t)} \frac{\partial \Psi(k, t)}{\partial t}+3 \alpha k^{5 / 3} \frac{\partial \Psi(k, t)}{\partial k}+2 \nu k^{2}=0 .
$$

The solution of eq. (10) along a characteristic is

$$
\Psi(k, t)=\frac{\Psi_{0}(k)}{k_{r}^{2}} \frac{k^{2}}{\left(1+2 \nu k^{2} t\right)},
$$

where $k_{r}$ defines the intersection point of the characteristic with the axis $t=$ 0 . The characteristics are parametrically defined by the implicit equation

$$
\frac{\Psi_{0}(k)}{k_{r}^{2}} \frac{k^{2}}{\left(1+2 \nu k^{2} t\right)}-\frac{\nu}{2 \alpha}\left(k_{r}^{4 / 3}-k^{4 / 3}\right)+\Psi_{0}(k)=0 ;
$$

each value of $k_{r}$ defines a specific characteristic. Since the curves defined by eq.(12) have a dependence of the type $t \propto 1 / k^{2}$, they define in the $k-t$ 
plane a family of hyperbolas having the axis $k=0$ as one of the asymptotes. This means that the spectrum will contract in time towards smaller wavenumbers, which is to be expected since energy is mostly dissipated in small ( $k \gg k_{0}$, where $k_{0}$ is the injection wavenumber) eddies. The initial condition $E_{0}(k)$ is the interpolation formula given by von Karman (1948) for the case of negligible viscosity, which not only reproduces the right $k^{-5 / 3}$ behavior of the spectrum in the inertial regime, but also in the small $k$ region, where it is known to be $\propto k^{4}$.

We can now compare the predictions of the present model with the degree of turbulence in the local ISM determined by the Linsky et al. (1993) experiment. The actual energy density inside the LB is $\epsilon_{t}=2.76 \times$ $10^{-12} \mathrm{erg} \mathrm{cm}^{-3}$ and it is presumably the same in the hot and in the neutral gas (Cox \& Reynolds 1987). If the explosion has taken place in a rarefied medium of density $n_{0} \sim 4 \times 10^{-3} \mathrm{~cm}^{-3}$, as suggested by Cox \& Reynolds (1987), and assuming for the total energy of the explosion $E=10^{51} \mathrm{ergs}$, then from case B of Chevalier (1974), the fraction $\eta$ of the total energy converted in kinetic energy is $\eta \sim 0.1$. Therefore, the kinetic energy density is $\epsilon_{k}=\eta \epsilon_{t} /(\chi-1)=3.06 \times 10^{-13} \mathrm{erg} \mathrm{cm}^{-3}$. This value can be used to fix the initial condition.

The time evolution of $E(k, t)$ is completely determined by the dynamic equation (9) when $k_{0}$, the energy injection wavelength, is specified. A considerable uncertainty remains in the determination of $k_{0}$ (for an instructive discussion see Spangler 1991); Spangler (1991) suggests $k_{0}=2 \pi / 10^{18} \mathrm{~cm}^{-1}$ as the most suitable value from observational arguments.

The most probable velocity $\xi_{k^{*}}$ measured at the time $t$ can be expressed in terms of the turbulent spectrum as

$$
\xi_{k^{*}}(t)=\left[\int_{k^{*}}^{\infty} d k E\left(k, t-t_{0}\right)\right]^{1 / 2}
$$

where $t_{0}$ is the time of the explosion, and $k^{*} \sim 2 \pi / d$, where $d$ is the distance on which the turbulence is sampled, in this case the distance to Capella $(d=13.2 \mathrm{pc})$.

The time evolution of the spectrum for $k_{0}=2 \pi 10^{-18} \mathrm{~cm}^{-1}$ is followed up $10^{7} \mathrm{yr}$ after the explosion, for a density $n=0.047 \mathrm{~cm}^{-3}$ and temperature $T=7000 \mathrm{~K}$ appropriate for the local HI (Linsky et al. 1993). In the first stage, lasting about $10^{5} \mathrm{yr}$, energy is dissipated only in small eddies $\left(k \gg k_{0}\right)$. An equilibrium spectrum is maintained through several wavelength decades while $k_{s}$ rapidly approaches the injection wavelength and the Reynolds number decreases accordingly; in this stage, however, $\xi_{k^{*}}$ decreases very slowly because most of the energy is stored in the large eddies with $k \sim k_{0}$. 
In the second stage, energy starts to be dissipated also in large eddies with $k \sim k_{0}$ at a rate $\propto t^{-2}$. At the end of the calculation $\left(\tau=10^{7} \mathrm{yr}\right)$, $\xi_{k^{*}}=0.039 \mathrm{~km} \mathrm{~s}^{-1}$.

The calculated $\xi_{k^{*}}$ can be compared with the observed one for different values of $k_{0}$ and $\tau$. If the explosion took place $2-10 \times 10^{5}$ yr ago (Cox \& Reynolds 1987), then a narrow range of outer scales satisfies the limits on $\xi_{k^{*}}$. This range is very much in agreement with the value suggested by Spangler (1991): $0.9 \times 10^{18} \lesssim \ell_{0} \lesssim 6 \times 10^{18} \mathrm{~cm}$; generally, it appears possible to consistently match the observed degree of turbulence in the local environment with the scenario of the LB created by a SN explosion. In fact, the use of $\tau=3.2 \times 10^{5} \mathrm{yr}$, suggested by Gehrels \& Chen (1993) from the "Geminga hypothesis", implies $\ell_{0}=1.5 \times 10^{18} \mathrm{~cm}$.

\section{Summary}

We have explored the effect of the photolevitation process, through which dusty diffuse clouds can rise to considerable high Galactic latitudes, on the global vertical distribution of the HI in the Galaxy. It is shown that the vertical support may be completely provided by turbulence in the lower effective gravitational potential determined by the Galactic radiation field. In addition, the apparent observed constancy of the HI scale height with galactocentric radius is naturally explained by the model in terms of the similar behavior of the Galactic radiative and gravitational fields.

We have investigated the origin and evolution of the turbulence taking the Local Bubble as a typical supernova-regulated environment. The evolution and decay of the turbulent spectrum injected by a supernova explosion in such an environment is studied through an analytical solution of the dynamic equation. The results, when compared with $H S T$ high resolution absorption line data, support the idea that the turbulent motions have been produced by a supernova explosion that occurred a few times $10^{5}$ years ago, as indicated also by other evidences.

\section{References}

Baker, P.L., \& Burton, W.B. 1979, $A \& A S, 35,129$

Batchelor, G. K. 1986, The Theory of Homogeneous Turbulence, (Cambridge: Univ. Press)

Bignami, G. F., Caraveo, P. A. \& Mereghetti, S. 1993, Nature, 361, 704

Bloemen, J.B.G.M. 1987, ApJ, 322, 694

Bruhweiler, F. C. \& Vidal-Madjar, A. 1987, Scientific Accomplishment of the IUE, ed. Y. Kondo, (Dordrecht:Reidel), 467

Boulares, A., \& Cox, D.P. 1990, ApJ, 365, 544

Chevalier, R. 1974, ApJ, 188, 501

Cox, D. P. \& Reynolds, R. J. 1987, $A R A \& A, 25,303$

Dickey \& Lockman 1990, ARA\&A, 28, 215

Ferrara, A., 1993, ApJ, 407, 157 
Ferrara, A, MNRAS, submitted

Franco, J., Ferrini, F., Ferrara, A., \& Barsella, B. 1991, ApJ, 366, 433

Gehrels, N. \& Chen, W. 1993, Nature, 361, 706

Heiles, C. 1991, in The Interstellar Disk-Halo Connection in Galaxies, ed. H. Bloemen, (Dordrecht:Kluwer), 433

Klein, R.I., McKee, C.F., \& Colella, P. 1990, in The Evolution of the Interstellar Medium, ed. L. Blitz, (San Francisco:PASP), 117

Kovasznay, L. S. G. 1948, J. Aeronaut. Sci., 15, 745

Kuijken, K., \& Gilmore, G. 1989, MNRAS, 239, 605

Lallement, R., Vidal-Madjar, A. \& Ferlet, R. 1986, $A \& A, 168,225$

Linsky, J. L., Brown, A., Gyley, K., Diplas, A., Savage, B. D., Aires, T. R., Landsman, W, Shore, S. N. \& Heap, S. R. 1993, ApJ, 402, 694

Lockman, F.J. 1984, ApJ, 283, 90

Lockman, F.J., \& Gehman, C.S. 1991, ApJ, 382, 182

McKee, C.F. 1990, in The Evolution of the Interstellar Medium, ed. L. Blitz, (San Francisco:PASP), 3

McKee, C. F. \& Ostriker, J. P. 1977, ApJ, 218, 148 (MO)

Schimdt, M. 1957, Bull. Astron. Inst. Neth, 13, 247

Savage, B.D., \& Massa, D. 1987, ApJ, 314, 380

Spangler, S. R. 1991, ApJ, 376, 540

von Karman, T. 1948, Proc. Natl. Acad. Sci. U.S., 34, 530

\section{DISCUSSION}

A. Whitworth: I recently estimated the mass-to-light ratio required for radiation pressure acting on dust to be comparable with gravity. If the gas is coupled to the dust, I obtain $(\mathrm{M} / \mathrm{L}) \sim 0.1\left(\mathrm{M}_{\odot} / \mathrm{L}_{\odot}\right)$ using standard dust parameters. Have you made such an estimate for your model?

Ferrara: I agree qualitatively with your estimation. The bulk of the support is provided by turbulence and radiation pressure contributes a 20\% fraction. However, the mass and light distribution in the Galaxy conspire, through the radiative support, to give a constant scale height of HI in spite of the change in the gravitational potential and turbulent properties of the ISM. 